\title{
A Ideografia do Tractatus Logico-Philosophicus e Funções Recursivas Primitivas
}

\section{Felipe Oliveira Araújo Lopes}

Universidade Federal de Minas Gerais

Departamento de Filosofia

Rua Dona Maria da Glória, 460, São Sebastião das Águas Claras, Nova Lima

Minas Gerais, Brazil

felipe.lopes99@yahoo.com.br

Received: 17.05.2016; Revised: 16.08.2016 Accepted: 26.08.2016

DOI: http://dx.doi.org/10.1590/0100-6045.2016.V39N2.FL

Resumo: No Tractatus Logico-Pbilosophicus (T) Wittgenstein apresenta o esboço de uma ideografia a qual ele pretende perspícua às condições essenciais de uma representação linguística. Seu estilo lacônico e sua notação elíptica, no entanto, deixam subentendidos diversos elementos fundamentais para o estabelecimento de uma sintaxe satisfatória com esse objetivo. O presente artigo faz o levantamento de alguns dos principais pontos obscuros em sua notação, buscando aproximá-la de estruturas similares às observadas em funções recursivas primitivas. A seção 1 discute como a lógica pura no Tractatus permite a obtenção de uma sintaxe lógica, comum a qualquer sistema de sinais em uma representação, e como a partir dela é possível estabelecer a ideografia. As seções 2 de 3 apresentam a derivação dessa ideografia utilizando os procedimentos da lógica pura, resultando na Forma Geral da Proposição, na Forma Geral da Operação, na Forma Geral da Série e na Forma Geral do Número. As seções 4 e 5 associam a notação ideográfica do Tractatus à notação de funções recursivo-primitivas.

Palavras Chave: Linguagem, Análise Lógica, Matemática, Recursão Primitiva.

Abstract: In the Tractatus Logico-Philosophicus (T) Wittgenstein presents a sketch of an ideography which he intends to be perspicuous to the essential conditions of a linguistic representation. His laconic style and concise notation, nevertheless, leaves implicit many elements that are fundamental for the establishment of a satisfactory syntax with that goal. The present article investigates some of the main obscure points in his

Manuscrito - Rev. Int. Fil., Campinas, v.39, n.2, pp. 59-88, abr.-jun. 2016. 
notation, searching for similarities with the structures usually seen in primitive recursive functions. Section 1 discusses how pure logic in the Tractatus allows the achievement of a logical syntax, essential to any system of signs in a representation, and how it is possible to establish an ideography from it. The sections 2 and 3 present the obtainment of his ideography by using procedures of pure logic, resulting in the General Form of the Proposition, the General Form of the Operation, the General Form of the Series and the General Form of the Number. Sections 4 and 5 associate the ideographic notation of the Tractatus with the notation of primitive recursive functions.

Keywords: Language, Logical Analysis, Mathematics, Primitive Recursion.

\section{Lógica Aplicada, Lógica Pura, Sintaxe Lógica e a Ideografia ${ }^{1}$}

O objetivo deste trabalho é investigar em detalhe a notação ideográfica introduzida por Wittgenstein nos aforismos T6, T6.01 e T6.02 do Tractatus Logico-Philosophicus, utilizando como referência comparativa o formalismo da teoria de recursões primitivas. Para tanto, a presente seção irá apresentar os pressupostos necessários à derivação de uma ideografia no contexto do Tractatus. Tais pressupostos dizem respeito ao ferramental analítico necessário a esse desenvolvimento, bem como ao fundamento dessas ferramentas nas condições mínimas necessárias ao estabelecimento de uma representação. Ainda nesta seção, justifica-se a Forma Geral da Proposição como parte da ideografia, e introduz-se o método de lógica pura a ser utilizado ao longo da seção 2, dedicada especificamente ao desenvolvimento ideográfico. A seção 3 apresenta uma contextualização dos resultados obtidos até então, enquanto a seção 4 realiza a comparação entre a notação de Wittgenstein e a de funções recursivas primitivas. Por fim, a seção 5 realiza um estudo de casos, comparando ambos os formalismos na obtenção da soma e do produto aritméticos.

$1 \mathrm{O}$ escopo do presente artigo se limita à recursão primitiva, e não à totalidade das funções computáveis, por essas últimas permitirem a construção do que poderiam ser chamadas de 'pseudoproposições indecidíveis', no contexto do período intermediário de Wittgenstein. Isso, no entanto, não tira o interesse de uma eventual avaliação da possibilidade de obtenção ou não de um correspondente para a $\mu$-recursão não limitada na notação do Tractatus.

Manuscrito - Rev. Int. Fil., Campinas, v.39, n.2, pp. 59-88, abr.-jun. 2016. 
Wittgenstein apresenta dois métodos de análise lógica no Tractatus; por meio da transformação de constantes em variáveis e por meio da reescrita de sinais proposicionais não analisados de modo às relações entre as condições de verdade das proposições serem expressas como tautologias e contradições. O primeiro método diz respeito à lógica pura, enquanto o segundo, à lógica aplicada. Ambos os métodos de análise encontram fundamento na concepção de que deve haver isomorfismo entre linguagem e mundo para que haja representação. No caso da lógica aplicada, é exatamente o isomorfismo necessário entre sinal proposicional não analisado e a situação relativamente complexa que ele representa o que demanda a possibilidade de uma análise desse sinal. Supondo $p$ um sinal proposicional não analisado, a sua estrutura, por definição, não expressa toda a complexidade da situação representada, de maneira a ser a sua complexidade sintática, dada por suas relações para com os demais sinais em um sistema, o que permite o isomorfismo entre $p$ e fatos no mundo. Tal complexidade sintática se manifesta em regras de combinação e substituição dos sinais envolvidos, e uma vez que a complexidade da situação representada é dada pelas condições de verdade das proposições em análise, necessariamente deve haver regras de substituição que permitam expressar, nos próprios sinais proposicionais quando analisados, as condições de verdade dessas proposições. Com isso a sintaxe dos operadores lógicos, ao refletir relações entre as condições de verdade das proposições, viabiliza a reescrita de sinais proposicionais de modo a explicitar a complexidade semântica dessas proposições em seus sinais mesmos.

A sintaxe dos operadores lógicos se mostra assim fundamental para o isomorfismo entre linguagem e mundo, sendo, portanto, parte da sintaxe lógica, essencial a qualquer sistema de sinais em uma representação. Mesmo proposições elementares - em que por definição não constam operadores lógicos - têm suas relações verdade para com outras proposições expressas por meio de operadores em tautologias e contradições. Dessa maneira, ainda que a lógica aplicada sirva à expressão das condições verdade de proposições particulares em seus sinais proposicionais, ela se vale de elementos sintáticos comuns a quaisquer proposições, sendo esses últimos propriamente o escopo de estudo da lógica pura. 
O método característico em lógica pura na explicitação das regras da sintaxe lógica é apresentado por Wittgenstein em T3.315 (a nota 2 a seguir aponta ainda um possível uso em lógica aplicada):

Se transformamos em variável uma parte constituinte de uma proposição, há uma classe de proposições que são todos os valores da proposição variável assim originada. Em geral, essa classe depende ainda do que nós, segundo uma convenção arbitrária, queremos significar com partes daquela proposição. Se transformamos em variáveis, porém, todos os sinais cujo significado foi arbitrariamente determinado, ainda assim continua a haver uma tal classe. Esta, porém, não depende mais de qualquer convenção, mas apenas da natureza da proposição. Ela corresponde a uma forma lógica - a um protótipo lógico de figuração.

A lógica aplicada se configura como um procedimento que conduz de termos previamente definidos àqueles termos indefiníveis, primitivos, de uma linguagem. Dentre os elementos indefiníveis que participam de uma proposição, têm-se aqueles que dizem respeito à proposição particular em caso (como os nomes que a compõem, por exemplo) e aqueles que dizem respeito à sintaxe de proposições quaisquer. O procedimento T3.315 fornece um método para a expressão da sintaxe desses elementos em geral2: ao transformar as constantes de um sinal proposicional em variáveis, obtemos como resultado esquemas da aplicação de tais regras sintáticas, ou 'protótipos lógicos'. É a partir das regras da sintaxe lógica, obtidas em lógica pura com a transformação de todas as constantes de um sinal proposicional em variáveis, que Wittgenstein propõe sua ideografia, pretendendo-a perspícua a essas formas lógicas. $\mathrm{O}$ isomorfismo

\footnotetext{
${ }^{2}$ Dada uma proposição $f a$, por exemplo, o procedimento permite obter $f x$ ao tornar a constante a variável. Assim, $f x$ reflete a sintaxe dos nomes que podem assumir a posição de $x$ nesse esquema. Essa deve ser considerada uma utilização do método T3.315 em lógica aplicada, portanto, por servir a expressar a sintaxe de sinais particulares. No entanto, ao tornar mesmo a constante $f$ variável, obtemos $\varphi x$, a qual indica a estrutura em argumento-função de proposições elementares quaisquer - e de onde a forma argumento-função ser parte da sintaxe lógica (sendo esse já um resultado em lógica pura). Naturalmente, proposições elementares poderiam ter outras posições de argumento além de $x$, o que implica nessas eventuais posições deverem ser consideradas subentendidas na variável $\varphi$, nesse exemplo.
}

Manuscrito - Rev. Int. Fil., Campinas, v.39, n.2, pp. 59-88, abr.-jun. 2016. 
entre linguagem e mundo, fundamento da representação, como visto, baseia-se na possibilidade de reescrita dos sinais proposicionais de modo a expressar as condições de verdade das proposições nesses sinais mesmos, com o uso de operadores lógicos. Assim, a Forma Geral da Proposição, resultado principal da análise em lógica pura, tem a seguinte estrutura (T6):

$$
[\bar{p}, \bar{\xi}, N(\bar{\xi})]
$$

Onde $\bar{p}$ indica o conjunto das proposições elementares, $N$ se trata de um operador lógico similar ao traço de Sheffer $^{3}$ e $[\bar{\xi}, N(\bar{\xi})]$ do esquema para a reiteração de $N$. Nessa expressão não se especifica qual a forma das proposições elementares $\bar{p}$, e tampouco o número ou a estrutura das reiterações de $N$, sendo essas as constantes tornadas variáveis na obtenção da Forma Geral da Proposição, através do procedimento T3.315. Nela somente se indica, com o uso do sinal de barra em $\bar{p}$ e $\bar{\xi}$, que um dos possíveis valores dessas variáveis deve se dar. Em repetidas aplicações de T3.315, Wittgenstein deriva então a Forma Geral da Operação, a Forma Geral da Série e a Forma Geral do Número, expressando-as em uma ideografia que se pretende perspícua a essas formas lógicas. Tal derivação é detalhada a seguir.

\section{Da Forma Geral da Proposição à Forma Geral do Número}

A lógica pura na derivação da ideografia do Tractatus leva de conceitos formais menos a mais abstratos, conforme sugerido pelo procedimento em T3.315. Da Forma Geral da Proposição, $[\bar{p}, \bar{\xi}, N(\bar{\xi})]$ (T) , chegamos à Forma Geral da Operação, $[\bar{\xi}, N(\bar{\xi})]^{\prime}(\bar{\eta})(=[\bar{\eta}, \bar{\xi}, N(\bar{\xi})])($ T6.01), e dessa à Forma Geral da Série em $\left[x, \xi, \Omega^{\prime} \xi\right]$ (onde $\Omega=[\bar{\xi}, N(\bar{\xi})]$ ) (T6.02). Essa última é reformulada com o uso de expoentes em $\left[\Omega^{0}{ }^{\prime} x, \Omega^{v^{\prime}} x, \Omega^{v+1} x\right]$ (T6.02)

\footnotetext{
3 Na notação apresentada por Hatcher (1968, p.10), o traço de Sheffer é o dual da operação $N$.
}

Manuscrito - Rev. Int. Fil., Campinas, v.39, n.2, pp. 59-88, abr.-jun. 2016. 
e dela, ao tornar variável mesmo a Forma Geral da Operação, $\Omega$, obtemos a Forma Geral do Número, $[0, \xi, \xi+1]$ (T6.03). Por essas estruturas decorrerem da sintaxe lógica, um agrupamento de sinais que não se enquadre nas regras determinadas por elas ou que não permita transformações sintáticas que resultem nelas não articula uma representação, mas nonsense. Assim, dado o aspecto recursivo dessas expressões, haveria um método para mecanicamente evitar a construção de contrassensos, bastando para tanto seguir um procedimento algorítmico na geração de sinais. Por outro lado, possíveis contrassensos poderiam ser identificados diretamente pela inspeção desses sinais, pela averiguação se se encontram ou não conforme os esquemas de substituição fornecidos por essas formas lógicas. Em outras palavras, não seria necessária uma transformação de sinais por meio de análise para validar a sua correção para com a sintaxe lógica, mas tão somente a conferência direta desses sinais mesmos, de onde essa se tratar de uma ideografia 4 .

Antes de passar ao detalhamento dessas fórmulas, é preciso esclarecer alguns dos elementos que as compõem, como as variáveis $\xi$ e $\bar{\xi}$ e o operador $N$. Acerca de $\xi$ e $\bar{\xi}$, tome-se T5.501:

Uma expressão entre parênteses cujos termos sejam proposições, indicoa - se a sequência dos termos entre os parênteses for irrelevante - por meio de um sinal da forma “( $\bar{\xi})$ ”. " $\xi$ ” é uma variável cujos valores são os termos da expressão entre parênteses; e o traço sobre a variável indica que ela substitui todos os seus valores entre parênteses. (Portanto, se $\xi$ tem, digamos, os 3 valores $\mathrm{P}, \mathrm{Q}$, R, então $(\bar{\xi})=P, Q, R$.). Os valores da variável são fixados. A fixação é a descrição das proposições que a variável substitui. (...) Podemos distinguir três espécies de descrição: 1. A

\footnotetext{
${ }^{4}$ A ideografia não se resume a esse conjunto de fórmulas, mas envolve ainda, por exemplo, a utilização de sinais distintos para símbolos distintos (e, conseqüentemente, nomes distintos para objetos distintos): "3.325 Para evitar esses equívocos, devemos empregar uma notação que os exclua, não empregando o mesmo sinal em símbolos diferentes e não empregando superficialmente da mesma maneira sinais que designem de maneiras diferentes. Uma notação, portanto, que obedeça à gramática lógica - à sintaxe lógica".
}

Manuscrito - Rev. Int. Fil., Campinas, v.39, n.2, pp. 59-88, abr.-jun. 2016. 
enumeração direta. Nesse caso, podemos simplesmente colocar, no lugar da variável, seus valores constantes. 2. A especificação de uma função $f x$, cujos valores para todos os valores de $x$ sejam as proposições a serem descritas. 3. A especificação de uma lei formal segundo a qual tais proposições sejam constituídas. Nesse caso, os termos da expressão entre parênteses são todos os termos de uma série formal.

A fixação de valores de uma variável em T5.501 se apresenta como o procedimento inverso ao de transformação de constantes em variáveis, em T3.315. Na fixação dos valores de $\xi$, temos que se $\xi=f x$, então $\bar{\xi}=(f a, f b, f c, \ldots) . \quad$ Em outras palavras, $\bar{\xi}$ equivale ao conjunto das proposições que podem ser valores da função ou variável proposicional $f x$, de onde se poder dizer que $\xi$ (nesse exemplo, $f x$ ) expressa a forma lógica comum a $f a, f b, f c, \ldots$. Além disso, se $\bar{\xi}$ é apresentada diante do sinal de uma operação, em sua posição de argumento, então as proposições $(\mathrm{P}, \mathrm{Q}, \mathrm{R})$ ou $(f a, f b, f c, \ldots)$, por exemplo, formam a base ou argumento dessa operação como se vê nas ocorrências do sinal da variável $\bar{\eta}$ na Forma Geral da Operação, $[\bar{\xi}, N(\bar{\xi})]^{\prime}(\bar{\eta})(=[\bar{\eta}, \bar{\xi}, N(\bar{\xi})])$. Por outro lado, se ao invés de $\bar{\xi}$ temos $\xi$ como base de uma operação ou argumento de função, como acontece na Forma Geral da Série e na Forma Geral do Número, $\left[x, \xi, \Omega^{\prime} \xi\right]$ e $[0, \xi, \xi+1]$, seu papel deve ser o de um esquema para a geração de expressões. Dessa maneira, a distinção entre $\bar{\xi}$ e $\xi$ deve ser tomada como a existente entre uma variável livre propriamente dita e uma letra esquemática - $\bar{\xi}$ possui um escopo fixo, trata-se de uma enumeração de proposições que perfaz uma totalidade sobre a qual podemos quantificar (ou aplicar uma operação lógica, como $N$ ), enquanto $\xi$ equivale a uma letra esquemática que se deixa substituir por expressões com determinada forma lógica. $\bar{\xi}$ é uma variável livre com um escopo delimitado, cujo esquema é dado por $\xi$.

Além disso, o termo $\bar{\xi}$ deve ser tomado como uma simples enumeração de sinais proposicionais - e não como uma proposição, como seria, por exemplo, o produto lógico das proposições correspondentes aos sinais proposicionais sob 
$\bar{\xi}$. Em outras palavras, em $\bar{\xi}$ não temos atribuições de valores verdade aos sinais listados, de modo que essa variável apenas reúne sob uma totalidade os sinais proposicionais com a forma do esquema $\xi$. Somente obtemos uma proposição após a aplicação de alguma operação lógica sobre $\bar{\xi}$, como em $N(\bar{\xi})$. De fato, dada uma lista de sinais proposicionais $(p, q, r)$, para obter uma proposição é preciso determinar ainda se as suas condições de verdade serão relacionadas por meio de uma conjunção, de uma disjunção, ou por condicionais, etc. Esse ponto se mostra útil ao esclarecer porque tautologias não afirmam nada acerca do mundo: por resultarem de operações que se anulam completamente, obtemos com elas variáveis livres, sinais desligados, exatamente como os que ocorrem na simples enumeração de sinais proposicionais em $\bar{\xi}$ antes da aplicação de alguma operação sobre eles.

O operador $N$, por sua vez, é uma generalização do traço de Sheffer, onde $N(p, q, r)$ equivale a $\sim p . \sim q . \sim r$ (T5.502), por exemplo. Em uma notação mais usual, escreveríamos nesse caso $(p|q| r)$, de maneira aos demais conectivos poderem ser definidos como $\sim p=(p \mid p)$; $p \cdot q=((p \mid p) \mid(q \mid q)) ; \quad p \vee q=((p \mid q) \mid(p \mid q)) ;$ etc. (T5.1311). Tal operador corresponde a uma generalização do traço de Sheffer por Wittgenstein pretender expressar com ele ainda o cálculo de predicados, e com isso a lógica de primeira ordem. Se por $\xi$ tomamos a variável proposicional $f x$, por exemplo, então $N(\bar{\xi})$ equivale à negação conjunta de todos os valores que essa variável pode assumir, em $\sim f a . \sim f b . \sim f c \ldots$. Nesse caso, $N(N(\bar{\xi}))$ equivaleria a $(\exists x) f x$ (T5.52). Por outro lado, o quantificador universal pode ser obtido da seguinte forma: se $\xi=N(f x)$, então $\bar{\xi}=\overline{N(f x)}=(\sim f a, \sim f b, \sim f c, \ldots)$, de maneira que $N(\bar{\xi})$ equivale a $\sim \sim f a . \sim \sim f b . \sim \sim f c \ldots=f a . f b . f c \ldots=(x) f x$. Assim, Wittgenstein pretende dar um tratamento uniforme ao cálculo proposicional e ao cálculo de predicados, ainda que ressalvas tenham sido feitas acerca da viabilidade dessa 
redução. Como observa Fogelin ${ }^{5}$, a múltipla quantificação seria inviável na notação do Tractatus, visto o operador $N$ manipular simultaneamente todas as posições de argumento de uma função, o que tornaria $(\exists x)(y) f x y$ inexprimível, por exemplo, assim como a lógica de primeira ordem. Geach propõe solucionar o problema estendendo a notação de Wittgenstein de maneira a cada operação $N$ apontar a posição de argumento específica à qual ela se aplica, através de um índice ${ }^{6}$. Independente dessa discussão, deve ser observado que ao barrar uma variável, como em $\bar{\xi}=(f a, f b, f c, \ldots)$, reunimos a totalidade dos termos com a forma geral $\xi=f x$. Assim, seria hipoteticamente possível reunir sob $\bar{\xi}$ infinitas expressões, caso existam infinitos objetos no mundo sob o escopo de $x$ em $f x$, algo que, posteriormente ao Tractatus, Wittgenstein irá rejeitar por equivaler a uma quantificação sobre infinitos termos.

Pode-se então esclarecer a Forma Geral da Proposição:

6 A forma geral da função de verdade é: $[\bar{p}, \bar{\xi}, N(\bar{\xi})]$. Isso é a forma geral da proposição.

${ }^{5}$ Fogelin, 1987, p.78-9.

${ }^{6}$ Geach, 1981, p. 169. Algo do tipo, no entanto, inviabiliza a distinção clara entre generalização e operações lógicas pretendida por Wittgenstein, visto que o uso de índices em $N$ para indicar a variável à qual o operador se aplica torna difusa essa distinção: "5.521 Separo o conceito todo da função de verdade. Frege e Russell introduziram a generalidade em conexão com o produto lógico ou a soma lógica. Assim, tornou-se difícil entender as proposições $(\exists x) \cdot f x$ e $(x) \cdot f x$, em que estão encerradas ambas as ideias". A esse respeito, ver nota 14, onde é discutida a oposição apresentada por Cuter entre operaçoes seletivas e operações construtivas. A totalização de um conjunto de sinais proposicionais seria realizada por operações formais seletivas, em contraste com operacooes lógicas, construtivas. A respeito do problema da múltipla quantificação em Wittgenstein, Landini apresenta uma solução aparentemente mais adequada em (Landini, 2007, p.134-146).

7 Wittgenstein aceita a possibilidade de infinitos objetos no Tractatus e, portanto, de infinitas proposições elementares, em T4.2211, sobre as quais podemos aplicar operações lógicas. Por outro lado, ele restringe o número de aplicações reiteradas de operações lógicas a uma quantidade finita (T5.32).

Manuscrito - Rev. Int. Fil., Campinas, v.39, n.2, pp. 59-88, abr.-jun. 2016. 
6.001 Isso nada diz senão que toda proposição é um resultado da aplicação sucessiva da operação $N(\bar{\xi})$ às proposições elementares.

6.002 Dada a forma geral como uma proposição é construída, com isso já está dada também a forma geral como, a partir de uma proposição e por meio de uma operação, uma outra pode ser gerada.

$\operatorname{Em}[\bar{p}, \bar{\xi}, N(\bar{\xi})], \quad \bar{p}$ equivale à fixação de um conjunto de proposições elementares, enquanto a expressão $[\bar{\xi}, N(\bar{\xi})]$ ao esquema da aplicação reiterada da operação $N(\bar{\xi})$ sobre essas proposições, onde a cada passo a expressão à esquerda, $\bar{\xi}$, se substitui pela expressão à direita, $N(\bar{\xi})$, gerando a série $\bar{\xi}, N(\bar{\xi}), N(N(\bar{\xi})), \ldots$ O esquema de reiteração é apresentado através de $[\bar{\xi}, N(\bar{\xi})]$ ela explicitamente exibir a base, $\bar{\xi}$, e o resultado, $N(\bar{\xi})$, de cada passo iterativo ${ }^{8}$. No caso, não é indicado o número específico de iterações realizadas, e também por isso essa se trata de uma forma geral. Dessa maneira, $[\bar{\xi}, N(\bar{\xi})]$ é a variável para a forma geral de uma operação resultante da composição de aplicações da operação $N$.

${ }^{8}$ É por meio do sinal $[\bar{\xi}, N(\bar{\xi})]$ que Wittgenstein pretende expressar, de maneira perspícua em sua ideografia, que "o que é comum às bases e ao resultado da operação são precisamente as bases" (T5.24) - algo que se vê claramente em $\bar{\xi}$ figurar tanto na primeira quanto na segunda posição de $[\bar{\xi}, N(\bar{\xi})]$. Por outro lado, esse sinal refletiria ainda que "a operação dá expressão à diferença das formas" (T5.24) - algo que se manifesta na diferença entre os sinais da base $\bar{\xi}$ e o do resultado $N(\bar{\xi})$ em cada iteração. Com isso Wittgenstein mostra, no próprio sinal ideográfico $[\bar{\xi}, N(\bar{\xi})]$ em T6.01, aquilo que aparentemente é 'dito' de maneira metalógica, e portanto nonsense, em T5.24. 
No entanto, para a viabilidade da notação proposta por Wittgenstein, $[\bar{\xi}, N(\bar{\xi})]$ deve ser entendida ainda não como simples iterações da operação $N$ sobre um conjunto de proposições elementares dadas ou sobre o resultado de cada aplicação a elas. Isso porque em cada iteração a fixação dos valores de $\bar{\xi}$ deve realizar uma nova seleção entre as proposições geradas até então, sem o que seria impossível gerar todas as proposições moleculares que podemos construir sobre uma base de proposições elementares $\bar{p}$. Digamos que o conjunto inicial de proposições elementares seja dado por $\bar{p}=(r, s, t)$. A primeira aplicação da operação $N$ resulta em $\left(\sim r_{.} \sim s . \sim t\right)$, enquanto uma segunda aplicação, realizada sobre esse resultado, obtém $\sim(\sim r . \sim s . \sim t)$. Já uma terceira aplicação, realizada sobre $\sim\left(\sim r_{.} \sim s_{.} \sim t\right)$, resulta $\mathrm{em} \sim \sim(\sim r . \sim s . \sim t)$, a qual equivale a $(\sim r . \sim s . \sim t)$, o mesmo resultado da primeira aplicação da operação. Qualquer outra iteração não nos leva mais longe, e sendo assim, por meio de simples reiterações sobre o resultado de uma mesma operação não seria possível gerar o conjunto das proposições moleculares que podemos construir a partir de $r, s$ e $\theta$. A solução seria considerar que a cada passo recursivo podemos realizar fixações distintas de valores em $\bar{\xi}$, tomando a cada vez diferentes subconjuntos de $\bar{p}$ ou das proposições geradas ao longo da série. Nesse caso, cada nova operação pode ser aplicada sobre um dos conjuntos $\bar{\eta}=(r), \bar{\theta}=(s), \bar{\psi}=(t), \bar{\sigma}=(r, s), \bar{\alpha}=(r, t), \bar{\beta}=(s, t)$, $\bar{\omega}=(r, s, t)$, ou algum outro subconjunto dentre as proposições geradas até então. Com isso, a cada nova iteração da operação $N$ é realizada uma nova fixação de $\xi$, o que gera, a cada passo, uma ramificaçãa ${ }^{10}$ distinta na série das

9 Poderíamos tentar considerar ainda que a fixação de $\bar{\xi}$ reúne todas as proposições geradas até então na série. Nesse caso, a primeira aplicação resulta em $(\sim r . \sim s . \sim t)$, a segunda em $\sim(\sim r . \sim s . \sim t)$ e a terceira em $\sim(\sim r . \sim s . \sim t) .(\sim r . \sim s . \sim t)$, uma contradição, o que igualmente é inaceitável por não permitir levar a série adiante na geração de todas as possíveis proposições moleculares baseadas em $r$, s e $t$.

10 Sobre o que se pretende por ramificação, aqui e no que segue, tomem-se as seguintes duas séries de sinais $(a, b, c, d, e, f, \ldots)$ e $(a, b, c, w, x, y, \ldots)$. Elas podem ser tomadas como Manuscrito - Rev. Int. Fil., Campinas, v.39, n.2, pp. 59-88, abr.-jun. 2016. 
proposições. A fixação dos valores de $\xi$ deve, portanto, se dar ao longo das aplicações de operações em $[\bar{\xi}, N(\bar{\xi})]$, se valendo de qualquer um dos procedimentos apresentados em T5.50111.

Assim, na avaliação de quais constantes foram tornadas variáveis na obtenção da Forma Geral da Proposição, $[\bar{p}, \bar{\xi}, N(\bar{\xi})]$, deve-se ter em conta que para obter uma instância dessa variável proposicional, ou seja, uma proposição, são fixados: i) os valores de $\bar{p}$, ii) o número de iterações $[\bar{\xi}, N(\bar{\xi})]$ e iii) as diferentes fixações de valores de $\xi$ em cada passo iterativo, na geração das diferentes ramificações da série de sinais. A fixação desses três itens se expressa na Forma Geral da Proposição pelo uso das variáveis barradas $\bar{p}$ e $\bar{\xi}$. Já em sentido inverso a essa 'síntese' de um sinal proposicional, seguindo o procedimento de análise T3.315 e dada uma proposição qualquer, ao tomar por variáveis os valores fixados nesses três itens obtemos novamente a Forma Geral da Proposição.

Em T6.002 Wittgenstein afirma que juntamente com a Forma Geral da Proposição já é introduzida uma Forma Geral da Operação ${ }^{12}$. Isso se justifica por uma nova aplicação do procedimento T3.315. No caso, a constante tornada variável diz respeito ao sinal do conjunto dos termos iniciais da série de proposições, o conjunto das proposições elementares $\bar{p}$. Com isso, operações

sendo uma só série, que se ramifica, ou bifurca, no ponto $c$. As duas ramificações obtidas nesse caso correspondem a duas fixações distintas de $\xi$ na quarta iteração de $N$.

11 Supondo a fixação de valores em cada passo sendo realizada pela aplicação de operações formais de seleção (ver nota 14), caso haja uma função proposicional fx com infinitos possíveis valores de argumento, devemos ter operações suficientes para selecionar $2^{\mathrm{N}}$ subconjuntos dentre o total das possíveis proposições formadas com esses nomes; o que demanda uma quantidade de operações necessárias de cardinalidade superior à dos números naturais. Essa linha de raciocínio pode ser útil para interpretar a posterior rejeição da possibilidade de infinitos argumentos em funções proposicionais por parte de Wittgenstein.

12 “6.002 Dada a forma geral como uma proposição é construída, com isso já está dada também a forma geral como a partir de uma proposição e por meio de uma operação, uma outra pode ser gerada."

Manuscrito - Rev. Int. Fil., Campinas, v.39, n.2, pp. 59-88, abr.-jun. 2016. 
aplicam-se a qualquer conjunto de proposições - e não apenas a proposições elementares - quando da fixação novamente do esquema $[\bar{\xi}, N(\bar{\xi})]$. O traço sobre uma variável proposicional como $\eta$ fixa seus valores em $\bar{\eta}$. Assim, o sinal de uma operação lógica antes de sua aplicação pode ser expresso por meio de $[\bar{\xi}, N(\bar{\xi})] \eta$ ou $[\bar{\xi}, N(\bar{\xi})](\eta)$ - ou ainda, $[\eta, \bar{\xi}, N(\bar{\xi})]$ - onde $\eta$ indica a posição no esquema $[\bar{\xi}, N(\bar{\xi})]$ obtida ao tornar $\bar{p}$ variável. Inversamente, aplicar a operação significa fixar esses valores, ao se tomar $[\bar{\xi}, N(\bar{\xi})]$ novamente no contexto de uma proposição, $[\bar{\eta}, \bar{\xi}, N(\bar{\xi})]$, de onde a Forma Geral da Operação ser dada por $[\bar{\xi}, N(\bar{\xi})]^{\prime}(\bar{\eta})(=[\bar{\eta}, \bar{\xi}, N(\bar{\xi})])$. Nessa expressão, o apóstrofo em $[\bar{\xi}, N(\bar{\xi})]^{\prime}$ corresponde à aplicação da operação $[\bar{\xi}, N(\bar{\xi})]$ fixando $\bar{\eta}$, em seu argumento, obtendo com isso o resultado $[\bar{\eta}, \bar{\xi}, N(\bar{\xi})]$, apresentado após o sinal de igualdade entre parênteses ${ }^{13}$. Aplicar uma operação não equivaleria, portanto, ao fragmento de sinal proposicional $[\bar{\xi}, N(\bar{\xi})] \eta$, antes de sua aplicação, nem à proposição $[\bar{\eta}, \bar{\xi}, N(\bar{\xi})]$, obtida após sua aplicação, mas à própria fixação dos valores de $\eta$ na variável operacional $[\bar{\xi}, N(\bar{\xi})] \eta$, expressa por meio do uso do apóstrofo. Será argumentado mais adiante que essa fixação de valores é ela mesma uma operação formal (ver nota 14). Com isso, a variável da Forma Geral da Operação é dada pelo sinal $[\bar{\xi}, N(\bar{\xi})]=\Omega$, ao passo que a expressão $[\bar{\xi}, N(\bar{\xi})]^{\prime}(\bar{\eta})(=[\bar{\eta}, \bar{\xi}, N(\bar{\xi})])$ equivale mais propriamente à aplicação

${ }^{13}$ Frascolla (1994, p.8-9) associa o apóstrofo ao uso desse mesmo sinal por Russell em funções descritivas. Em Russell, dada uma relação $a \mathrm{R} b$, temos que $(a \mathrm{R})^{\prime}=b$. No entanto, nesse caso o apóstrofo se aplica sobre uma expressão incompleta, (aR), tendo por resultado um nome, $b$, enquanto aqui ele se aplica sobre um conjunto de sinais proposicionais, tendo por resultado uma proposição. O uso do apóstrofo proposto por Russell em descrições definidas equivale a um caso particular de aplicação de uma operação formal no contexto do Tractatus, ao passo que, na introdução da Forma Geral da Operação, acima, Wittgenstein lida com operações lógicas (ver nota 14). O uso do apóstrofo pode ser interpretado de maneira idêntica em ambos os casos.

Manuscrito - Rev. Int. Fil., Campinas, v.39, n.2, pp. 59-88, abr.-jun. 2016. 
dessa operação, ao fixar o esquema $\eta$ em $\bar{\eta}$. Cuter (2005, p.69-70) chega a uma conclusão similar, ao afirmar que "operações [de seleção] como $O_{i}$ e $O_{i i}$ encontram-se subentendidas em variáveis proposicionais como $\xi$ e $\eta$ " (comentário meu entre colchetes) ${ }^{14}$.

A Forma Geral da Série $\left[x, \xi, \Omega^{\prime} \xi\right]$ pode ser obtida em uma nova aplicação do procedimento T3.315. Nesse caso, da Forma Geral da Proposição $[\bar{p}, \bar{\xi}, N(\bar{\xi})]$ não apenas $\bar{p}$ é tornada uma variável não barrada (resultando $\eta$ na Forma Geral da Operação, acima, e $x$ no que segue), como também $\bar{\xi}$, obtendo $\xi^{15}$. Dessa maneira, de $[\bar{p}, \bar{\xi}, N(\bar{\xi})]$ passamos à Forma Geral da Operação $[\eta, \bar{\xi}, N(\bar{\xi})]$ e dessa última a $\left[x, \xi, \Omega^{\prime} \xi\right]$, a Forma Geral da Série, na qual $\Omega$ é a forma geral de uma operação lógica qualquer $[\bar{\xi}, N(\bar{\xi})]$. Foi visto que variáveis barradas como $\bar{\xi}$ expressam a fixação de $\xi$, em $\bar{\xi}=(f a, f b, f c)$, por exemplo, ao passo que variáveis não barradas como $\xi$ expressam a forma comum a tais valores, como em $\xi=f x$. Assim, variáveis barradas delimitam uma totalidade específica de expressões, ao passo que

14 Operações formais de seleção devem ser entendidas em contraste com operações lógicas, ou de construção (Cuter, 2005, p.69): “A construção de proposições complexas a partir de proposições simples é sempre um processo duplo. Primeiro, uma seleção é realizada por um dos três procedimentos em T5.501. Em segundo, a operação $N$ é aplicada às proposições selecionadas." (itálico meu). É à distinção entre operações formais e operações lógicas a que Wittgenstein se refere em T5.521: "Separo o conceito todo da função de verdade". As primeiras permitiriam realizar totalizaçôes de conjuntos de sinais proposicionais, ao passo que as últimas tão somente aplicariam operações verdade sobre esses totais, projetando seus sinais proposicionais de acordo com as condições de verdade da proposição molecular assim composta. A distinção entre operações formais de totalização e operações lógicas de construção é o que permitiria evitar a definição de um "conjunto de todos os conjuntos que não pertencem a si mesmos", no paradoxo de Russell.

15 Ao tornar uma variável a base de proposições elementares $\bar{p}$, temos que operações podem ser aplicadas a partir de qualquer ponto da série de proposições, assim como séries podem se iniciar em qualquer ponto da série de proposições.

Manuscrito - Rev. Int. Fil., Campinas, v.39, n.2, pp. 59-88, abr.-jun. 2016. 
variáveis não barradas deixam em aberto esse total, por exprimirem tão somente a forma comum aos termos em questão. No caso da Forma Geral da Proposição, o uso de $\bar{\xi}$ no esquema de reiteração $[\bar{\xi}, N(\bar{\xi})]$ indica uma totalidade específica de iterações e, portanto, uma posição específica na série de sinais proposicionais. Já na Forma Geral da Série, o uso de $\xi$ no esquema de reiteração $\left[\xi, \Omega^{\prime} \xi\right]$ não indica um total, mas a possibilidade potencialmente infinita de gerar novos termos na série, cuja forma geral é dada pela regra de substituição de $\xi$ por $\Omega^{\prime} \xi$ a cada passo em $\xi, \Omega^{\prime} \xi, \Omega^{\prime} \Omega^{\prime} \xi, \Omega^{\prime} \Omega^{\prime} \Omega^{\prime} \xi, \ldots$. Em outras palavras, na apresentação da Forma Geral da Proposição, foi argumentado que a cada passo na aplicação reiterada de $N$ em $[\bar{\xi}, N(\bar{\xi})]$ devemos fixar novamente os valores de $\bar{\xi}$, o que resulta em uma ramificação especifica dentre as possíveis na geração da série das proposições, bem como um número específico de iterações. Ao tornar variável mesmo a fixação de valores indicada pelas barras em $[\bar{\xi}, N(\bar{\xi})]$, resultando em $[\xi, N(\xi)]$, não chegamos à forma geral de uma posição particular na série lógica ${ }^{16}$, dada pela Forma Geral da Proposição, nem à forma geral de posições relativas entre proposições em séries lógicas, dada pela Forma Geral da Operação, mas sim à forma de séries lógicas em geral: $\left[x, \xi, \Omega^{\prime} \xi\right]$ é a forma comum às possíveis séries geradas por operadores lógicos $\Omega$ quaisquer.

Com isso, na Forma Geral da Série já deve haver uma variável numérica implícita em $\left[\xi, \Omega^{\prime}(\xi)\right]$, a qual é tornada explícita por Wittgenstein em sua segunda versão da Forma Geral da Série, com expoentes, em $\left[\Omega^{0}{ }^{\prime} x, \Omega^{v^{\prime}} x, \Omega^{v+1^{\prime}} x\right]$, obtida a partir de $\left[x, \xi, \Omega^{\prime} \xi\right]$ por meio das definições $x=\Omega^{0^{\prime}} x$ Def. e $\Omega^{\prime} \Omega^{v^{\prime}} x=\Omega^{v+1^{\prime}} x$ Def. (T6.02). Uma vez expressa a Forma Geral da Série em $\left[\Omega^{0} x, \Omega^{v^{\prime}} x, \Omega^{v+1} x\right]$, através de nova aplicação do procedimento T3.315 chega-se à Forma Geral do Número $[0, \xi, \xi+1]$, onde a constante tornada variável é a própria Forma Geral da Operação, $\Omega$. A variável operacional $\Omega$ tem por valores operações verdade - ou operações lógicas -

16 Entendendo séries lógicas, geradas por operações lógicas, em contraste com séries formais, geradas por operações formais.

Manuscrito - Rev. Int. Fil., Campinas, v.39, n.2, pp. 59-88, abr.-jun. 2016. 
resultantes da composição de aplicações reiteradas da operação $N$ sobre uma variável proposicional. Ao se tornar variável mesmo o sinal $\Omega$ na Forma Geral do Número, tem-se que números determinam regras da sintaxe lógica para a aplicação operações em geral, e não somente operações lógicas. Em outras palavras, a sintaxe dos números constitui regras que refletem o caráter recursivo da aplicação de operações quaisquer na transformação de sinais.

No entanto, duas dificuldades se apresentam para essa interpretação: A) Em T6.02, Wittgenstein claramente identifica a fórmula $\left[x, \xi, \Omega^{\prime} \xi\right]$ com a série $x, \Omega^{\prime} x, \Omega^{\prime} \Omega$ 'x $\Omega^{\prime} \Omega^{\prime} \Omega^{\prime} x . .$. O que diferencia a Forma Geral da Série, assim expressa, da Forma Geral da Proposição, $[\bar{p}, \bar{\xi}, N(\bar{\xi})]$, ou seja, da forma geral de um termo na série, é o uso de variáveis barradas nessa última, a indicar a fixação de $p$ e $\xi$ sob uma totalidade qualquer. Dessa maneira, a Forma Geral da Série expressa a possibilidade potencialmente infinita de expansão de uma série qualquer, por se valer da letra esquemática não barrada $\xi$. No entanto, a fórmula $[0, \xi, \xi+1]$ igualmente não utiliza variáveis barradas, o que deveria indicar a forma da série dos números naturais, ao passo que o intuito explícito de Wittgenstein é expressar a Forma Geral do Número (T6.03) - a forma geral de um termo na série dos naturais. Nesse contexto, uma formulação mais coerente com a presente leitura seria $[0, \bar{\xi}, \bar{\xi}+1]$. B) Wittgenstein introduz o sinal $\Omega=[\bar{\xi}, N(\bar{\xi})]$ como a Forma Geral da Operação. Apesar disso, foi argumentado acerca da necessidade da existência de operaçôes formais para que a notação do Tractatus possa atender o intuito da construção de uma ideografia. Wittgenstein dá indicações de reconhecer essa necessidade. Ao apresentar pela primeira vez o conceito de operação em T5.2521, por exemplo, ele utiliza a fórmula $\left[a, x, O^{\prime} x\right]$. Seria contrário à própria ideografia o uso de sinais distintos para um mesmo símbolo, caso com o uso de $O$ se pretendesse a variável posteriormente utilizada para operações lógicas, $\Omega$. Além disso, a letra ' $a$ ' nessa formulação é utilizada ao longo de todo o Tractatus para expressar nomes, ao passo que operações lógicas apenas se aplicam a proposições ${ }^{17}$. No próprio

\footnotetext{
${ }^{17}$ Novos problemas se apresentam com essa observação, visto operações deverem se aplicar a sinais quaisquer, e não apenas a nomes ou proposições. A mesma dificuldade ocorre com o uso do termo $x \mathrm{em}\left[x, \xi, \Omega^{\prime} \xi\right]$, tendo em vista que ao longo do livro $x$ é uma variável para nomes, ao passo que operações lógicas apenas se aplicam a Manuscrito - Rev. Int. Fil., Campinas, v.39, n.2, pp. 59-88, abr.-jun. 2016.
} 
Tractatus são apresentadas ainda séries formais que não poderiam ser obtidas com funções verdade, como a Série Sucessor, em T4.1273, onde a cada etapa em $a R b, \quad(\exists x): a R x . x R b, \quad(\exists x, y): a R x . x R y . y R b, \ldots$, são introduzidas novas variáveis $x, y, z, \ldots$, não havendo indício de como a sintaxe de $N$ poderia realizar algo do tipo. Sendo assim, uma interpretação para $\Omega=[\bar{\xi}, N(\bar{\xi})]$ poder ser considerada a Forma Geral da Operação é com ela Wittgenstein estar apresentando propriamente parte da sua ideografia, na qual necessariamente devem constar operações lógicas, ao passo que as operações formais particulares necessárias à descrição do mundo diriam respeito a uma questão de lógica aplicada'18.

\section{A Matemática e A Lógica como Estrutura da Linguagem}

Tomando a estrutura de sistemas de sinais em geral, como apresentada na seção anterior, em analogia com a topologia de um grafo, proposições corresponderiam aos vértices, enquanto operações às arestas que ligam esses vértices. Séries, por sua vez, delimitam diferentes percursos pelo grafo, e enfim, os pontos de cruzamentos entre séries mostram como elas se entrelaçam, articulando um sistema de sinais. Tais pontos de cruzamento entre séries podem por sua vez ser expressos por meio de identidades entre seus termos, as quais, por remeterem às posições numéricas de cada termo em sua respectiva série, assumem a forma de equacõoes matemáticas. Passamos, com isso, do aspecto mais local ao mais amplo na topologia de um sistema de sinais, mas não obtemos uma hierarquia entre essas formas lógicas, visto elas se encontrarem sobrepostas

proposições. Por razões como essas talvez não seja possível encontrar coerência completa na notação do Tractatus, mas tão somente buscar preencher as lacunas deixadas pelas indicações feitas no livro, como tentado aqui.

18 Toda operação lógica deve ser considerada uma operação formal, no sentido de que também realiza transformações de sinais, ainda que o contrário não se dê. Sendo assim, operações lógicas fazem parte do escopo da lógica pura, ao passo que operações apenas formais como as que refletem a sintaxe dos nomes dizem respeito à lógica plicada (operações formais referentes à sintaxe dos nomes são tais que geram, a partir de $f x$, a expressão $f a$, por exemplo).

Manuscrito - Rev. Int. Fil., Campinas, v.39, n.2, pp. 59-88, abr.-jun. 2016. 
na Forma Geral da Proposição - e assim também em qualquer proposição particular. A Forma Geral da Proposição deve, portanto, indicar, através da estrutura de seu próprio sinal, a sua posição em um sistema de linguagem, valendo-se para tanto de conceitos formais que exprimam essa posição, como os de operação, série, número, etc. Assim, para determinar uma posição em um sistema proposicional devemos fixar um conjunto de proposições elementares, a operação sendo reiterada, a série em que se dá a reiteração - ou seja, a sequência particular de ramificações ou bifurcações ao longo das iterações - e o número dessas iterações. Feito isso, os pontos de cruzamento entre as diversas séries na articulação desse sistema podem ser expressos por meio de equações, com as quais realizamos cálculos com sinais em geral.

A matemática deve assim ser tomada como parte da sintaxe lógica, por refletir regras no cálculo de operações com sinais quaisquer. Que $2+3=5$, por exemplo, indica que a aplicação reiterada de 2 operações, seguida de outras 3 aplicações dessa mesma operação, obtém o mesmo sinal que 5 aplicações dessa operação sobre a mesma base. Visto a sintaxe dos números aplicar-se a operações quaisquer, ela é igualmente válida para operações lógicas, de onde a correspondência entre equações e tautologias em T4.461 e T6.12119. Algo do tipo, no entanto, não implica em uma maior generalidade da matemática em relação à lógica (em um logicismo às avessas ${ }^{20}$, em uma redução da lógica à matemática), mas sim decorre de ambas refletirem diferentes aspectos de um sistema de sinais, de uma sintaxe para a transformação de sinais na constituição

19 “4.461 Tautologia e contradição não são, porém, contrassensos; pertencem ao simbolismo, analogamente à maneira, na verdade, como o " 0 " pertence ao simbolismo da aritmética.”; “6.121 As proposições da lógica demonstram as propriedades lógicas das proposições, ao ligá-las em proposições que não dizem nada. Esse método poderia também chamar-se um método-zero.”. Assim como operações lógicas podem se anular em uma tautologia, equações matemáticas sempre podem ser escritas como uma identidade a zero.

20 A matemática entendida como recurso linguístico descartável no resultado de uma análise completa das proposições é recorrente em leituras logicistas do Tractatus. Certamente podemos realizar transformações com operações sem nos valermos de números, mas o resultado dessas transformações necessariamente se encontra de acordo com o obtido por vias numéricas. Equações matemáticas não são assim consequências acidentais de nossas transformações com sinais, mas a expressão de um aspecto essencial a elas.

Manuscrito - Rev. Int. Fil., Campinas, v.39, n.2, pp. 59-88, abr.-jun. 2016. 
de uma representação. O caráter a priori da matemática e da lógica decorre assim de suas regras refletirem a sintaxe lógica necessária a qualquer representação, algo que igualmente justifica a sua aplicação universal nos diversos ramos das ciências exatas. Da mesma maneira, explica-se ainda a rejeição da existência de objetos matemáticos ou lógicos, visto regras da matemática e da lógica serem tão somente reflexos da própria estrutura da linguagem na representação do mundo e seus objetos.

\section{Funções Recursivas Primitivas e a Ideografia do Tractatus}

A associação entre elementos da ideografia do Tractatus e a teoria da recursão tem óbvio interesse em vista do finitismo e do construtivismo cada vez mais acentuados no pensamento posterior de Wittgenstein ${ }^{21}$. Na teoria da recursão primitiva, seguindo a notação de Boolos (1984, p.74-6), há três tipos de funções recursivas iniciais - assim chamadas por não serem compostas de nenhuma outra - e duas operações ${ }^{22}$, a serem aplicadas um número finito de vezes na obtenção das demais funções recursivas primitivas. São as funções iniciais:

Zero: $z(x)=0$ para todo $x \in N$;

Sucessor: $s(x)=x+1$ para todo $x \in N$; e

Projeção: $i d_{i}^{n}\left(x_{1}, \ldots, x_{i}, \ldots, x_{n}\right)=x_{i}$.

As seguintes operações, por sua vez, permitem a definição de funções recursivas umas a partir das outras:

Composição: $h\left(x_{1}, \ldots, x_{n}\right)=f\left(g_{1}\left(x_{1}, \ldots, x_{n}\right), \ldots, g_{m}\left(x_{1}, \ldots, x_{n}\right)\right)$, ou $C n[f, g]$;

Recursão Primitiva: $h\left(x_{1}, \ldots, x_{n}, 0\right)=f\left(x_{1}, \ldots, x_{n}\right) \mathrm{e}$

$h\left(x_{1}, \ldots, x_{n}, s(y)\right)=g\left(x_{1}, \ldots, x_{n}, y, h\left(x_{1}, \ldots, x_{n}, y\right)\right)$, ou $b=\operatorname{Pr}[f, g]$.

21 Assim como certamente há interesse na aproximação com os formalismos do lambda-calculus e o de gramáticas gerativas, dada a concepção algorítmica de linguagem do filósofo.

22 O contexto no que segue deverá deixar claro se o termo “operação" é utilizado na acepção do Tractatus ou se na da teoria da recursão.

Manuscrito - Rev. Int. Fil., Campinas, v.39, n.2, pp. 59-88, abr.-jun. 2016. 
Por simplicidade, no que segue a variável em negrito $\boldsymbol{x}$ deve ser entendida como representando o vetor $x_{1}, \ldots, x_{n}$, em $h(\boldsymbol{x}, 0)=f(\mathbf{x})$ e $h(\boldsymbol{x}, \boldsymbol{s}(y))=g(\boldsymbol{x}, y, h(\mathbf{x}, y))$.

A função zero pode ser emulada na notação do Tractatus pela definição de uma operação $\theta$ que anule qualquer sequência de aplicações de outras operações. Supondo $O$ uma variável para operações quaisquer, a definição de $\theta$ é $\theta^{\prime}\left(O^{\prime}(x)\right)=O^{o^{\prime}}(x)=x$ def. No caso particular de operações lógicas, tem-se $\theta^{\prime}\left(\Omega^{\prime}(x)\right)=N^{0^{\prime}}(x)=x$ def. Uma vez que números apenas ocorrem em expoentes de operações, na prática a aplicação da operação $\theta$ equivale à função inicial $z(y)=0$ para qualquer natural $y$.

A função inicial sucessor encontra paralelo imediato na série formal sucessor, apresentada por Wittgenstein em T4.1273 (seção 2). No entanto, deve-se ainda verificar até que ponto os postulados de Peano podem ser considerados subentendidos no esquema $\left[a, x, O^{\prime} x\right]$ ou na Forma Geral do Número, $[0, \xi, \xi+1]$, uma vez que os postulados resultam em uma definição dos números naturais. São eles (seguindo Hatcher, 1968, p.70, com ligeiras modificações):

0 é um número natural;

(ii) para cada número natural $n$, existe exatamente um número natural $s(n)$, chamado sucessor de $n$;

(iii) $\quad 0$ não é o sucessor de nenhum número natural;

(iv) números naturais diferentes têm sucessores diferentes (função 1-1);

(v) todo conjunto que contenha 0 e o sucessor de quaisquer números contidos nele contém todos os números naturais (números naturais são o menor conjunto satisfazendo essas 4 propriedades).

(i) O primeiro postulado pode ser considerado implícito no fato de toda série gerada por operações na notação do Tractatus possuir um termo inicial, como ' $a$ ', em $\left[a, x, O^{\prime} x\right]$, quando da não aplicação de nenbuma operação - ou de zero operações em $[0, \xi, \xi+1]$. (ii) $O$ segundo postulado se reflete no uso da variável não barrada $x$ em $\left[a, x, O^{\prime} x\right]$ - ou ainda, $\xi$, em $[0, \xi, \xi+1]$ a exprimir a possibilidade sempre aberta de reiterar a operação, gerando um novo termo na série. Esse postulado determina ainda um caráter funcional para o sucessor, algo que encontra correspondente na série $\left[a, x, O^{\prime} x\right]$, dado que a aplicação da operação $\left[x, O^{\prime} x\right]$ sobre um termo $y$ obtém um novo sinal unívoco, $O^{\prime} y$. $\mathrm{O}$ mesmo vale para $[0, \xi, \xi+1]$. (iii) $\mathrm{O}$ terceiro postulado não 
se aplica ao esquema $\left[a, x, O^{\prime} x\right]$, uma vez que operações podem se anular e com isso o termo $a$ eventualmente se repetir na série gerada. Números, no entanto, enquanto expoentes de operações em $[0, \xi, \xi+1]$, não se anulam quando da reiteração dessas últimas, mas são incrementados em cada nova aplicação. Assim, em uma operação particular $O$, definida como $O^{\prime} O^{\prime} x=x$, é a operação que se anula, e ainda que em consequência dessa definição tenhamos $O^{\prime} O^{\prime} O^{\prime} x=O^{\prime} x$, numericamente ocorrem expoentes distintos em cada lado dessa identidade. Com isso, a regra de anulação que determina a sintaxe de $O$ atua como um mapeamento entre os possíveis expoentes dessa operação. No exemplo, a aplicação reiterada de $O$ sobre $x$ gera em seu expoente a série $0,1,2,3, \ldots$, como em qualquer operação arbitrária, mas uma vez dada a definição particular de $O$ - em $O^{\prime} O^{\prime}(x)=x-$ obtemos um mapeamento, uma função da série $0,1,2,3 \ldots$ à série $0,1,0,1, \ldots$. (iv) $\mathrm{O}$ quarto postulado pode ser escrito como 'se $x \neq y$, então $s(x) \neq s(y)$ ', onde $s$ é a função sucessor. O mesmo argumento utilizado em (iii) se aplica aqui. A reiteração de uma operação sobre bases distintas $x$ e $y$ pode eventualmente resultar em um mesmo valor, dada a possibilidade de operações se anularem, algo que contraria $s(x) \neq s(y)$. No entanto, a aplicação reiterada de operações não anula os expoentes numéricos, mas sim realiza um mapeamento entre possíveis valores desses expoentes. (v) $\mathrm{O}$ quinto postulado de Peano delimita uma totalidade dos números naturais. A princípio ele não seria contemplado pela notação do Tractatus, visto séries serem introduzidas por letras esquemáticas não barradas, como $\xi$; e esquemas de iteração, isoladamente, não determinam totalidades. No entanto, em T5.501 Wittgenstein afirma para casos similares que ao barrar $\xi$ obtemos "todos os termos de uma série formal" (meu itálico). Por outro lado ainda, foi argumentado que a fixação de $\xi$ implica fixar uma variável numérica implícita nesse esquema iterativo, de onde essa fixação não resultar na totalidade da série, mas sim na totalidade de termos dessa série gerados por certo número, finito, de operações. Independentemente dessa discussão, (v) não participa da definição da função sucessor - em jogo aqui - mas da concepção clássica dos números naturais como perfazendo uma totalidade infinita. A concepção finitária certamente é atendida pelo esquema $[0, \xi, \xi+1]$.

Manuscrito - Rev. Int. Fil., Campinas, v.39, n.2, pp. 59-88, abr.-jun. 2016. 
Funções iniciais de projeção selecionam um valor dentre um conjunto de argumentos dados ${ }^{23}$, em um papel análogo ao uso pretendido para a barra em $\bar{\xi}$, ou às operações formais de seleção propostas por Cuter, conforme discutido na seção 2. A diferença para com o Tractatus se encontra no sinal da barra em $\bar{\xi}$, conforme T5.501, dever se aplicar a variáveis proposicionais não ordenadas, ao passo que os argumentos $x_{1}, \ldots, x_{n}$ em $i d_{i}^{n}\left(x_{1}, \ldots, x_{i}, \ldots, x_{n}\right)=x_{i}$ são ordenados de 1 a $n$.

No que diz respeito às operações utilizadas para definir funções recursivas primitivas umas a partir das outras, a operação de composição é dada por $\quad C n[f, g]=h\left(x_{1}, \ldots, x_{n}\right)=f\left(g_{1}\left(x_{1}, \ldots, x_{n}\right), \ldots, g_{m}\left(x_{1}, \ldots, x_{n}\right)\right)$. Essa operação tem paralelo no Tractatus em definições como $\psi^{\prime}(x)=\theta^{\prime}\left(O_{m}{ }^{\prime}(x)\right)$, onde $\theta, \psi$ e $O_{m}$ são operações quaisquer, de modo a $h$ corresponder à operação sendo definida $\psi, f$ corresponder a $\theta, O_{m}$ às funções $g_{1}, \ldots, g_{m}$ e $x$ ao conjunto de argumentos $x_{1}, \ldots, x_{n}$. Pelo procedimento T3.335, tomando-se isoladamente $\theta$ e $O_{m}$, obtemos as formas $\theta(\eta)$ e $O_{m}(\xi)$, em que a fixação de $\xi$ em $\bar{\xi}$ determina os argumentos $x_{1}, \ldots, x_{n}$ e a fixação de $\eta$ em $\bar{\eta}$ determina os argumentos $g_{1}, \ldots, g_{m}$.

Por fim, a operação de recursão primitiva tem a forma $h(\boldsymbol{x}, 0)=f(\mathbf{x})$ e $h(\boldsymbol{x}, s(y))=g(\boldsymbol{x}, y, h(\mathbf{x}, y))$ - ou $h=\operatorname{Pr}[f, g]$. A primeira dessas equações é a base de recursão, o primeiro termo da série gerada, enquanto a segunda o passo recursivo utilizado na geração de novos termos na série. A base de recursão comparece na notação de Wittgenstein como o termo inicial de séries em geral. Já no passo recursivo, a principal característica que garante a expressividade da função $h \mathrm{em}$ definição é seu valor para o argumento $(\boldsymbol{x}, s(y))$ ser dado a partir de uma função $g$, a qual contém em seu argumento $\boldsymbol{x}$ (os parâmetros da recursão), y (o argumento de $h$ na iteração anterior) e $h(\boldsymbol{x}, y)$ (o resultado de $h$ na iteração

${ }^{23}$ Assim, é possível obter todos os subconjuntos de um conjunto de argumentos utilizando a composição $C n[f, g]=h\left(x_{1}, \ldots, x_{n}\right)=f\left(g_{1}\left(x_{1}, \ldots, x_{n}\right), \ldots, g_{m}\left(x_{1}, \ldots, x_{n}\right)\right)$, onde $g_{1}$ a $g_{m}$ são funções projeção $i d_{i}^{n}$ distintas entre si e $m \leq n$. Fazendo $m$ variar de 0 a $n$, temos uma estrutura recursiva que permite tomar como argumento de $f$ qualquer dos subconjuntos de $x_{1}, \ldots, x_{n}$.

Manuscrito - Rev. Int. Fil., Campinas, v.39, n.2, pp. 59-88, abr.-jun. 2016. 
anterior). Os argumentos $x_{1}, \ldots, x_{n}=\boldsymbol{x}$ foram anteriormente vistos terem como correspondentes a fixação de valores em $\bar{\xi}$ no Tractatus. Já o restante da estrutura do passo recursivo em $h(\boldsymbol{x}, s(y))=g(\boldsymbol{x}, y, h(\boldsymbol{x}, y))$ será discutida a seguir.

Tome-se a aplicação reiterada de uma operação $[\bar{\xi}, N(\bar{\xi})]$ sobre um conjunto de proposições elementares iniciais $\bar{p}$. É no procedimento de geração dessa série onde se deve buscar identificar, aproximadamente, expressões correspondentes a $h(\boldsymbol{x}, s(y)) ; h(\boldsymbol{x}, y) ; g(\mathbf{x}, y, h(\mathbf{x}, y)) ; s(y)$ e $y$. A primeira aplicação da operação resulta na proposição $q=[\bar{p}, \bar{\xi}, N(\bar{\xi})]$, a segunda em $r=[\bar{q}, \bar{\xi}, N(\bar{\xi})]$, e assim sucessivamente. Realizando a substituição de $q$ na expressão de $r$, obtemos $r=[[\bar{p}, \bar{\xi}, N(\bar{\xi})], \bar{\xi}, N(\bar{\xi})]$. Essa última expressão pode ser reescrita como $r=[\bar{p}, \bar{\eta}, N(\bar{\eta})]$, onde se substituiu a variável $\bar{\xi}$ por $\bar{\eta}$, para indicar que a operação $[\bar{\xi}, N(\bar{\xi})]$ aplicada para obter $r$ a partir de $q$ é distinta da operação $[\bar{\eta}, N(\bar{\eta})]$, utilizada para obter $r$ a partir de $\bar{p}$. No caso, $[\bar{\eta}, N(\bar{\eta})]=[\bar{\xi}, N(\bar{\xi})]^{2}$, de modo que $r$ pode ser reescrita como $\left.r=\left[\bar{p},[\bar{\xi}, N(\bar{\xi})]^{2}\right)\right]$. Considere-se agora uma apresentação alternativa de $r$ como $r=\left[\left[\bar{p},[\bar{\xi}, N(\bar{\xi})]^{1}\right], \bar{\xi}, N(\bar{\xi})\right]$. Substituindo essas duas últimas expressões uma na outra temos:

$$
\left.\left[\bar{p},[\bar{\xi}, N(\bar{\xi})]^{2}\right)\right]=\left[\left[\bar{p},[\bar{\xi}, N(\bar{\xi})]^{1}\right], \bar{\xi}, N(\bar{\xi})\right]
$$

Na identidade acima, a fórmula $\left.\left[\bar{p},[\bar{\xi}, N(\bar{\xi})]^{2}\right)\right]$ como um todo pode ser associada a $h(\boldsymbol{x}, s(y))$, na qual o expoente 2 corresponde a $s(y)$. Na fórmula à direita da identidade, a operação $[\bar{\xi}, N(\bar{\xi})]$ na parte mais externa corresponde à função $g$ repetidamente aplicada em cada etapa recursiva - e a qual aqui se aplica sobre $\left[\bar{p},[\bar{\xi}, N(\bar{\xi})]^{1}\right]$, o correspondente ao resultado no passo recursivo anterior $h(\mathbf{x}, y)$, e sobre o expoente 1 , correspondente ao argumento $y$. Ainda que a associação dessa maneira esboçada diga respeito a uma única iteração na passagem de $y=1$ a $y=2$, ela pode ser igualmente efetuada utilizandose $y$ e $s(y)$ no lugar de 1 e 2 , bem como com um esquema $\eta$ no lugar do 
conjunto inicial de proposições elementares $\bar{p}$, completando assim a analogia para com o passo $h(\boldsymbol{x}, s(y))=g(\mathbf{x}, y, h(\mathbf{x}, y))$. Temos como resultado:

$$
\left.\left[\eta,[\bar{\xi}, N(\bar{\xi})]^{s(y)}\right)\right]=\left[\left[\eta,[\bar{\xi}, N(\bar{\xi})]^{y}\right], \bar{\xi}, N(\bar{\xi})\right],
$$

em outra versão para a Forma Geral da Operação. Levando em conta ainda a possibilidade da existência de operações formais - para além de operações lógicas - essa fórmula se reescreve como:

$$
\left.\left[x,[\bar{\xi}, O(\bar{\xi})]^{s(y)}\right)\right]=\left[\left[x,[\bar{\xi}, O(\bar{\xi})]^{y}\right], \bar{\xi}, O(\bar{\xi})\right] .
$$

\section{Somas e Produtos Aritméticos - Estudo de Caso}

Nesta seção são comparados os tratamentos da soma e da multiplicação na ideografia do Tractatus e na notação da teoria da recursão. Abaixo, o exemplo de multiplicação no Tractatus, conforme a interpretação de Frascolla (1994, p.15-19), com algumas modificações para manter a notação original de Wittgenstein. À demonstração de $2 \times 2=4$ de Wittgenstein (T6.241) ele acrescenta ainda a regra $[\mathrm{A}]$, conforme a seguir:

$$
\begin{array}{ll}
{[1] \Omega^{(2 \times 2)^{\prime}} x=\left(\Omega^{2}\right)^{2^{\prime}} x} & \text { [por definição em } \Omega^{v \times \mu^{\prime}} x=\left(\Omega^{v}\right)^{\mu^{\prime}} x, \text { T6.241] } \\
{[2]=\left(\Omega^{2}\right)^{1+1^{\prime}} x} & \text { [pela definição do numeral 2 em T6.02] } \\
{[3]=\Omega^{2^{\prime}} \Omega^{2^{\prime}} x} & \text { [pela definição } \Omega^{\prime} \Omega^{v^{\prime}} x=\Omega^{v+1^{\prime}} x \text { em T6.02, } \\
{[4]=\Omega^{1+1^{\prime}} \Omega^{1+1^{\prime}} x} & \text { com } \Omega^{2} \text { no lugar de } \Omega \text { ] } \\
{[5]=\left(\Omega^{\prime} \Omega^{\prime}\left(\Omega^{\prime} \Omega\right)^{\prime} x\right.} & \text { [pela definição do numeral 2, T6.02] } \\
{[6]=\left(\Omega^{\prime} \Omega\right)^{\prime} \Omega^{\prime} \Omega^{\prime} x} & \text { [a ser tratado na discussão abaixo] } \\
{[7]=\Omega^{\prime} \Omega^{\prime} \Omega^{\prime} \Omega^{\prime} x} & \text { [pela definição da regra A] } \\
{[8]=\Omega^{1+1+1+1^{\prime}} x} & \text { [pela definição da regra A] } \\
{[9]=\Omega^{4} x} & \text { [pela definição indutiva em T6.02] } \\
{[\mathrm{A}] \quad\left(\Omega^{\prime} \Omega\right)^{\prime} \xi=\Omega^{\prime} \Omega^{\prime} \xi} & \text { [pela definição do numeral 4] }
\end{array}
$$


A passagem de [4] a [5] a princípio não parece se justificar, segundo Frascolla (1994, p.15), visto que utilizando a definição $x=\Omega^{0^{\prime}} x$ Def. e $\Omega^{\prime} \Omega^{v^{\prime}} x=\Omega^{v+1^{\prime}} x$ Def. em T6.02, deveríamos ter algo como $\Omega^{0+1+1^{\prime}} \Omega^{0+1+1^{\prime}} x=\Omega^{0+1+1^{\prime}} \Omega^{\prime} \Omega^{\prime} x$. No entanto, deve-se observar a ausência do 0 no expoente em $\Omega^{1+1^{\prime}} \Omega^{1+1^{\prime}} x$, o que impede a aplicação de $x=\Omega^{0} x$. Assim, o desenvolvimento seria $\Omega^{1+1^{\prime}} \Omega^{1+1} x=\left(\Omega^{1+1}\right)^{\prime}\left(\Omega^{1+1}\right)^{\prime} x=\left(\Omega^{\prime} \Omega^{1}\right)^{\prime}\left(\Omega^{\prime} \Omega^{1}\right)^{\prime} x=\left(\Omega^{\prime} \Omega\right)^{\prime}\left(\Omega^{\prime} \Omega\right)^{\prime} x$.

Dessa maneira, é a passagem de [1] para [2] na transformação $2=1+1$ que aparentemente contradiz a definição original do numeral $2 \mathrm{em} \mathrm{T6.02,} \mathrm{dada} \mathrm{por}$ $0+1+1=2$ Def., por descartar o número 0 inicial. Uma saída interpretativa seria considerar que Wittgenstein está introduzindo mais um aspecto de sua notação, na qual a ausência do zero em $1+1 \mathrm{em}\left(\Omega^{2}\right)^{1+1^{1}} x$ indicaria esse se tratar do expoente de $\left(\Omega^{2}\right)$, ao invés de novas iterações de $\Omega$. Já o sinal $\Omega^{2}$ entre parênteses nessa expressão não possui apóstrofo, o que igualmente impede a aplicação da definição $x=\Omega^{0^{*}} x$, e decorre diretamente da definição $\Omega^{v \times \mu^{\prime}} x=\left(\Omega^{v}\right)^{\mu^{\prime}} x$. Por outro lado, o passo [3] aparenta realmente se tratar de um equívoco, por nele nada indicar algum uso do numeral 2 distinto de sua definição original. Enfim, o passo [6] não consta no Tractatus, mas é introduzido por Frascolla quando afirma não haver como explicar a passagem direta de [5] para [7] senão por intermédio de uma regra como [A]. No entanto, o exemplo utilizado por Wittgenstein talvez não seja o mais adequado, visto o resultado de $2 \times 2$ ser o mesmo que $2+2$, o que não permite destacar claramente as diferenças entre ambas. Tome-se o caso $2 \times 3$, o qual se escreve por $\left(\Omega^{\prime} \Omega\right)^{\prime}\left(\Omega^{\prime} \Omega^{\prime} \Omega\right)^{\prime} x$. A ser utilizada a regra $[\mathrm{A}]$ de Frascolla $-\left(\Omega^{\prime} \Omega\right)^{\prime} \xi=\Omega^{\prime} \Omega^{\prime} \xi$ - temos que $\left(\Omega^{\prime} \Omega\right)^{\prime}\left(\Omega^{\prime} \Omega^{\prime} \Omega\right)^{\prime} x=\Omega^{\prime} \Omega^{\prime} \Omega^{\prime} \Omega^{\prime} \Omega^{\prime} x$; um resultado obviamente incorreto, visto $2 \times 3 \neq 5$.

Segue uma tentativa de solução para esse problema utilizando alguns dos elementos notacionais discriminados ao longo deste artigo. Foi argumentado que, dada a operação $\Omega=[\bar{\xi}, N(\bar{\xi})]$, o uso do apóstrofo deve ser interpretado como uma fixação dos possíveis valores desse esquema. Em outras palavras, o papel do apóstrofo é fixar, ou barrar, a variável $\eta$ na posição 
de argumento do sinal da operação $[\bar{\xi}, N(\bar{\xi})] \eta$, de modo a obter a variável proposicional $[\bar{\eta}, \bar{\xi}, N(\bar{\xi})]$. No entanto, não somente a posição de argumento da operação é uma variável no esquema $[\bar{\xi}, N(\bar{\xi})]$, como também o número de iterações de $N$, ou o expoente numérico de $N$, na constituição da operação composta $\Omega$. Assim, reescrevendo $\left(\Omega^{\prime} \Omega\right)^{\prime}$ como $\left(\Omega^{\prime} \Omega \eta\right)^{\prime}$, o produto $\left(\Omega^{\prime} \Omega\right)^{\prime}\left(\Omega^{\prime} \Omega^{\prime} \Omega\right)^{\prime} x$ pode ser reescrito como $\left(\Omega^{\prime} \Omega \eta\right)^{\prime}\left(\Omega^{\prime} \Omega^{\prime} \Omega \xi\right)^{\prime} x$. A ausência de apóstrofo nas operações compostas dentro dos primeiros parênteses deve ser entendida como indicando que o apóstrofo externo a eles não está fixando o valor do argumento $\eta$, mas sim o valor da variável numérica subentendida em cada um dos $\Omega$ 's entre parênteses. Esse valor é fixado pelo número de operações da expressão que se encontra na posição de argumento da variável operacional $\left(\Omega^{\prime} \Omega \eta\right)^{\prime}$, a saber, $\left(\Omega^{\prime} \Omega^{\prime} \Omega \xi\right)^{\prime}$, de modo a obter $\left(\Omega^{\prime} \Omega^{\prime} \Omega^{\prime} \Omega^{\prime} \Omega^{\prime} \Omega \xi\right)^{\prime} x$. Novamente, há uma operação sem apóstrofo entre parênteses nesse resultado. Apesar disso, pode-se supor ainda que caso $x$ seja a expressão de uma operação, a multiplicação deve prosseguir, mas caso ela seja fixada em uma variável proposicional, a única variável passível de ser tornada constante por esse termo é a posição de argumento $\xi$, de onde $\Omega^{\prime} \Omega^{\prime} \Omega^{\prime} \Omega^{\prime} \Omega^{\prime} \Omega^{\prime} x$, em $2 \times 3=6$ iterações de $\Omega$. Já o caso $2+3=5$ pode ser obtido diretamente em $\left(\Omega^{\prime} \Omega^{\prime} \eta\right)^{\prime}\left(\Omega^{\prime} \Omega^{\prime} \Omega^{\prime} \xi\right)^{\prime} x=\Omega^{\prime} \Omega^{\prime} \Omega^{\prime} \Omega^{\prime} \Omega^{\prime} x$, com a substituição de $\left(\Omega^{\prime} \Omega^{\prime} \Omega^{\prime} \xi\right)$ em $\eta$ e de $x$ em $\xi$, como indicada pelo apóstrofo à esquerda dessas letras esquemáticas entre parênteses. Dessa maneira, e em contraste com a definição do produto em $\Omega^{v \times \mu^{\prime}} x=\left(\Omega^{v}\right)^{\mu^{\prime}} x$, seria possível sugerir uma definição similar para a soma utilizando $\Omega^{v+\mu^{\prime}} x=\left(\Omega^{v^{\prime}}\right)^{\mu^{\prime}} x$. Wittgenstein, no entanto, introduz o sinal de soma juntamente com os números naturais, sem uma definição à parte para ele, como será mencionado mais adiante.

O que distingue a soma da multiplicação nesses exemplos é qual a variável a ser fixada em $\Omega^{\prime} \Omega$. No caso de $\left(\Omega^{\prime} \Omega^{\prime} \eta\right)^{\prime} x$, a posição de argumento $\eta$ é fixada, resultando na soma, ao passo que em $\left(\Omega^{\prime} \Omega \eta\right)^{\prime} x$ fixase a variável numérica subentendida em $\Omega$, em uma multiplicação. Como definições recursivas podem ser novamente efetuadas na definição da 
potenciação, da super-potenciação, etc., é preciso avaliar como se dão essas formas de fixação no contexto do esquema geral $\left.\left[\bar{\eta},[\bar{\xi}, N(\bar{\xi})]^{s(y)}\right)\right]=\left[\left[\left[\bar{\eta},[\bar{\xi}, N(\bar{\xi})]^{y}\right], \bar{\xi}, N(\bar{\xi})\right]\right.$, apresentado ao final da última seção, tendo em vista a sua correspondência para com o passo recursivo $h(\mathbf{x}, s(y))=g(\mathbf{x}, y, h(\mathbf{x}, y))$. Tome-se primeiramente o caso particular das definições da soma e do produto na teoria da recursão ${ }^{24}$ :

$$
\begin{gathered}
\operatorname{sum}(x, 0)=i d(x)=x, \operatorname{sum}(x, s(y))=s(\operatorname{sum}(x, y)) ; \\
\operatorname{prod}(x, 0)=z(x)=0, \operatorname{prod}(x, s(y))=\operatorname{sum}(x, \operatorname{prod}(x, y)) ;
\end{gathered}
$$

A soma é obtida utilizando $g=s$ e $b=$ sum em $h(\boldsymbol{x}, s(y))=g(\mathbf{x}, y, h(\mathbf{x}, y))$, assim como $\operatorname{sum}(x, 0)=i d(x)$ no lugar de $h(\boldsymbol{x}, 0)=f(\boldsymbol{x})$. No caso, a cada incremento da variável $y$, a operação $g$, ou seja, $s$, é aplicada novamente sobre o resultado de $h$, ou sum, da etapa anterior. Em outras palavras, na definição de $b$ (ou sum) a variável $y$ atua como o expoente de $g$ (ou s). Analogamente, na multiplicação temos $g=$ sum e $b=$ prod; e a variável $y$ como expoente de sum. Assim, em uma notação menos formal, a operação de recursão primitiva pode ser entendida como definindo o expoente de uma função: a função sum estabelece um expoente $y$ para a função sucessor $s$, em $s(x)$, bem como a função prod estabelece um expoente para a função $\operatorname{sum}$, em $\operatorname{sumv}^{y}(x, 0)$. No caso, a fórmula $s^{y}(x)$ claramente equivale à soma $x+y$ para números naturais $x$ e $y$ quaisquer, ao passo que, na fórmula $\operatorname{sunv}^{\prime}(x, 0)$, deve-se considerar subentendido que o resultado de cada aplicação de sum deve ser substituído na segunda posição de argumento de $\operatorname{sum}(x, \xi)$ na iteração subsequente, tendo 0 como valor inicial da série. A substituição de um desses resultados no esquema $\operatorname{sum}(x, \xi)$ equivale à fixação do valor de $\xi$ em $\bar{\xi}$ na notação do Tractatus, de modo que o valor de $\xi$ tornase totalmente fixo quando ambas as variáveis numéricas $x$ e $y$ são fixadas. Um raciocínio similar se aplica a a $s^{\prime}(x)$, tendo por esquema de iteração $s(\xi)$ em cada passo: fixar $\xi$ nessa expressão equivale a fixar os valores de $x$ e $y$ em $s^{y}(x)$, tendo $O$ como valor inicial da série. Em ambos os casos temos o equivalente a "fixar a posição de argumento $\eta$ ", na explicação apresentada acima acerca da

24 Boolos (1989, p.76), ou de maneira alternativa, sum $=\operatorname{Pr}\left[i d, C n\left[s, i d_{3}^{3}\right]\right]\left(x_{1}, x_{2}, x_{3}\right)$ e prod $=\operatorname{Pr}\left[r_{3} \operatorname{Cn}\left[\operatorname{sum}_{1}, i_{1}^{3}, i d_{3}^{3}\right]\right]\left(x_{1}, x_{2}, x_{3}\right)$ (idem).

Manuscrito - Rev. Int. Fil., Campinas, v.39, n.2, pp. 59-88, abr.-jun. 2016. 
soma e do produto no Tractatus. Por outro lado, a fixação de $g$ em $s$ ou em sum determina se a função sendo definida se trata da soma ou da multiplicação, respectivamente. A fixação de $g$ seria o equivalente a "fixar a variável numérica subentendida em $\Omega$ ”, na discussão acima.

Raciocínio similar deve se aplicar na comparação do passo recursivo $h(\boldsymbol{x}, s(y))=g(\boldsymbol{x}, y, h(\mathbf{x}, y)) \quad$ com a forma geral $\left.\left[\bar{\eta},[\bar{\xi}, N(\bar{\xi})]^{s(y)}\right)\right]=\left[\left[\left[\bar{\eta},[\bar{\xi}, N(\bar{\xi})]^{y}\right], \bar{\xi}, N(\bar{\xi})\right]\right.$. O equivalente à função $h(\mathbf{x}, y)$ definida por recursão primitiva é a expressão $\left[\left[\bar{\eta},[\bar{\xi}, N(\bar{\xi})]^{y}\right]\right.$, e equivalendo às posições de argumento $\boldsymbol{x}$ e $y$ temos $\bar{\eta}$ e $y$, respectivamente. À função $g$, sendo reiterada em cada passo recursivo e tendo $y$ por expoente, corresponde $[\bar{\xi}, N(\bar{\xi})]$. Com isso, o ato de fixar a posição de argumento em $\left(\Omega^{\prime} \Omega^{\prime} \eta\right)^{\prime} x$ pode ser considerado análogo a fixar os valores de $\boldsymbol{x}$ e de $y \mathrm{em}$ $h(\mathbf{x}, s(y))=g(\mathbf{x}, y, h(\mathbf{x}, y))$; ou ainda, a fixar os valores de $\bar{\eta}$ e $y$ em $\left[\left[\bar{\eta},[\bar{\xi}, N(\bar{\xi})]^{y}\right]\right.$ - Por outro lado, fixar a variável numérica subentendida em $\Omega$ pode ser considerado similar a fixar a função $g$ - a qual pode ser uma função recursiva inicial, bem como uma composição ou uma função recursiva de outras recursivo-primitivas, de maneira análoga a como $\Omega$ expressa uma composição de aplicações de $N$ qualquer. Caso $g$ resulte na função sucessor $s$ quando dessa fixação, a função $b$ sendo definida é a soma, caso $g$ seja a soma, então $b$ é a multiplicação, e assim sucessivamente, para a potenciação, super-potenciação, etc.

Por fim, há um problema proeminente na comparação entre as duas notações. A recursão primitiva permite definir expoentes de funções, algo que no Tractatus é realizado por meio de $x=\Omega^{0^{\prime}} x \quad$ Def. e $\Omega^{\prime} \Omega^{v^{\prime}} x=\Omega^{v+1^{\prime}} x \quad$ Def. (T6.02). A analogia entre essas definições e $h(x, 0)=f(x)$ e $h(\boldsymbol{x}, s(y))=g(\boldsymbol{x}, y, h(\mathbf{x}, y))$, no entanto, é problemática. Wittgenstein introduz a soma juntamente com os números naturais, ao passo que na teoria da recursão ela somente pode ser definida após a definição de sucessor, a qual primeiramente introduz os naturais. Uma definição alternativa seria $x=\Omega^{0^{\prime}} x$ Def. e $\Omega^{\prime} \Omega^{v^{\prime}} x=\Omega^{s(v)^{\prime}} x$ Def., onde $s$ é o sucessor. Nesse caso, (i) $h(\boldsymbol{x}, s(y))$ corresponderia a $\Omega^{s(v)^{\prime}} x$; (ii) $h(\boldsymbol{x}, y)$ a $\Omega^{v^{\prime}} x$; (iii) $g$ a $\Omega^{\prime}$, em $\Omega^{\prime} \Omega^{v^{\prime}} x$ (com essa última fórmula correspondendo a $g(\boldsymbol{x}, y, h(\boldsymbol{x}, y))$ ); (iv) y a $v$; (v) $\boldsymbol{x}$ a $x$ (ou à fixação de $x$ ); de modo análogo às associações realizadas na discussão anterior.

Manuscrito - Rev. Int. Fil., Campinas, v.39, n.2, pp. 59-88, abr.-jun. 2016. 


\section{Conclusões}

A dificuldade de traçar paralelos entre a ideografia do Tractatus e funções recursivas primitivas decorre, naturalmente, da diversidade de elementos deixados subentendidos por Wittgenstein em sua notação. No entanto, a estrutura iterativa da Forma Geral da Operação, a ocorrência mesma de uma Forma Geral da Série na ideografia, bem como a interpretação de números como expoentes de operações, indicam claramente sua intenção original de retratar estruturas recursivas. Em particular, no que diz respeito a números como expoentes de operações, a analogia se dá para com o fato de funções recursivas primitivas com a forma $h(\boldsymbol{x}, s(y))=g(\mathbf{x}, y, h(\boldsymbol{x}, y))$ resultarem na variável de recursão, $y$, equivaler ao expoente de reiteração da função $g$, utilizada para definir $h$. No entanto, e em contraste, como observado ao final da seção 5 , na teoria da recursão números naturais são definidos através da função sucessor, a qual, por sua vez, é utilizada na definição da operação de recursão primitiva - não sendo os naturais, portanto, introduzidos juntamente com essa última. As consequências formais e filosóficas dessa postura de Wittgenstein deverão ser avaliadas em outra oportunidade. De qualquer maneira, a analogia com a fórmula $h(\boldsymbol{x}, s(y))=g(\boldsymbol{x}, y, h(\boldsymbol{x}, y))$ tomando-se $y$ como expoente de $g$ parece corroborar a posição defendida por autores como Hylton (2008, p.138-152), os quais entendem as operações formais do Tractatus como correspondentes a funções matemáticas em geral (abrangendo funções de sinais quaisquer, e não apenas de números). Por fim, deve-se observar ainda que ao incluir a Forma Geral do Número em sua notação Wittgenstein aparenta ter em vistas uma aplicação ideográfica específica: o uso da matemática na física e demais ciências exatas. Notações cujas constantes numéricas se apresentam explicitamente nos sinais proposicionais permitem a sua manipulação de maneira perspícua para com as definições desses sinais - ou ainda, para com os "acordos tácitos" em que a sua sintaxe se baseia (T4.002). A sintaxe dos números, expressa em equações, reflete o caráter recursivo das operações lógicas e formais utilizadas nessas definições, ao mesmo tempo em que demandam uma quantidade reduzida de termos na expressão de sequências extensas de sinais. As proposições utilizadas na física e demais ciências exatas são, assim, proposições moleculares não analisadas, mas escritas em uma notação ideográfica, matemática, e com isso de maneira transparente às definições recursivas de seus termos, permitindo transformações adequadas para com elas. Assim, a 
matemática não somente reflete a estrutura recursiva da linguagem, de onde seu caráter a priori, conforme mencionado na seção 3 , como ainda viabiliza o estabelecimento de notações eficientes, desde o ponto de vista de sua capacidade expressiva. Essa seria a explicação para a proficuidade dos diversos ramos matematizáveis do discurso científico.

\section{Referências}

Boolos G., Jeffrey, R. Computability and Logic. Cambridge University Press, 3rd Edition, 1989.

Cuter, J. "Operations and Truth Operations in the Tractatus". Philosophical Investigations, vol.28, p.63-75, 2005.

FogELIN, R. Wittgenstein. Routledge, 1987.

FrasCOLLA, P. Wittgenstein's Philosopby of Mathematics. Routledge, 1994.

Frege, G. "On Concept and Object". Mind, vol.60, no 238, p. 168-180. Oxford University Press, 1951.

GEACH, P. “Wittgenstein's Operator N”. Analysis, vol.41, no.4, p.168-171. Oxford University Press, 1981.

GOLDFARB, W. 'Logic in the Twenties: The Nature of the Quantifier". The Journal of Symbolic Logic, vol.44, no. 3, p.351-368. Association for Symbolic Logic, 1979.

HatcheR, W. Foundations Of Mathematics. Saunders Company, 1968.

HyLton, P. "Propositions, Functions and Analysis”, p.138-152. Oxford, 2008.

LANDINI, G. 'Wittgenstein's Apprenticeship with Russell', p.134-146 . Cambridge, 2007.

WitTGensteIn, L. Tractatus Logico-Philosophicus. Repr. 1994. Editora USP, 1918. 